\title{
The Darulshafa of Astan Quds Razavi: A Pioneer in Women Treatment
}

\section{A R T I C L E I N F O}

\section{Article Type}

Historical Research

\section{Authors}

Karimian A.* MSc

\section{How to cite this article} Karimian A. The Darulshafa of Astan Quds Razavi: A Pioneer in Women Treatment. Sarem Journal of Reproductive Medicine. 2017; 1(1):21-28
*Sarem Cell Research Center (SCRC), Sarem Women's Hospital, Tehran, Iran

${ }^{1}$ Medicine Department, Medicine Faculty, Tehran University of Medical Sciences, Tehran, Iran

\section{Correspondence}

Address: Sarem Women's Hospital, Basij Square, Phase 3, Ekbatan Town, Tehran, Iran. Postal Code: 1396956111

Phone: +98 (21) 44670888

Fax: +98 (21) 44670432

ali_karimiyan_2011@yahoo.com

\section{Article History}

Received: August 21, 2015

Accepted:December 24,2015

ePublished: February 15, 2017

\section{A B S T R A C T}

Introduction Evidence indicates that it has long been considered public health and the observance of pilgrims and the poor people near the Haram of Imam Reza, sometimes it was called "Darulshafa", and the founders were those good people who were trying to provide as much as possible the facilities for the welfare of sick and poor people.

Conclusion In Teimori period, Goharshad Khatun founded a Darulshafa nearby the Haram at the time of establishing the Goharshad Mosque. In addition, in Safavieh period there used to be other Darulshafa which received several devotions which the most apparent one was Atighi devotion. In Afshar period, Adelshah repaired Imam Reza Darulshafa and devoted part of his property to it. In Ghajarieh period, Mirza Jafarkhan Moshiroldoleh founded a new type of Darulshafa. After the First World War, Dr. Amir Alam in his trip to Mashhad by presenting a report to Ahmadshah, took action to fundamental reforms in the organization and income of Darulshafa. He also intended to isolate the male and female section of Darulshafa completely. In 1933, Imam Reza Hospital was established by Mohammad Ali Asadi and its second head of the hospital was Professor Hammer Shelak who himself was a obstetrician. Since 1937, the Nursing School was established under the supervision of foreign nurses in this hospital until it was handed over to Iranian nurses in 1941. Now Astan Quds Razavi by having 1048 endowments is one of the biggest endowments in Islamic world.

Keywords Darulshafa; Astan Quds Razavi; Women; Imam Reza Hospital

\section{I T A T I O N L I N K S}

[1] Hospital and Dar al-Shafa in the holy shrine of Razavi in the historical mirror [2] Javid Armaghan, or life of the nestes of Dr [3] History of Iran's Hospital (from beginning to today) [4] Iran under the Safavavids [5] Razavi Guest house from Safavid to Ghajar Period (Volume I) [6] National library and archives of Iran. Accession No. 23525 [7] Dastoor Al Molouk Mirza Rafia [8] Dastoor Al Molouk Mirza Rafia va Tazkarat Al Moluok e Mirza Samiea [9] Dehkhoda Dictionary (Volume I) [10] Landlord and peasant in Persia [11] The face of the sacred endowment Astan Moghaddas Samen Alaemah Ali Ibn Musa al Reza [12] Terminology of law [13] Matla Al Shams [14] Presentation of Shrine documents and associated places in the Qajar period [15] Ferdows al tavarikh [16] Permanent memories: Astan Quds dar al Shafa narrates the documents [17] Treatise on research on the crossroad [18] Story of the Nawab Toluity of the magnificent Astan Quds Razavi [19] Orduye Homayouni newspaper [20] Khorasan travel newspaper [21] Azadi histoty [22] Seyf Al Doleh travelogue [23] Memories of Hajj Sayyah [24] Second travelogue of Khorasan [25] Orduye Homayoon newspaper [26] Khorasan trip newspaper with Naser Al Din Shah [27] Iran newspaper [28] Khurasan and Sistan [29] Mashhad from the tourists' view from 1600-1914 [30] Adab newspaper [31] Foyoozat: Head of the office of the endowment on endowments [32] A look at Waqf and its economic and social effects [33] Astan Qods Razavi foundation after mashroote revolution [34] A Look at traditional medicine in the Dar ul Shafi Astan Quds Razavi in the Safavid age (Review of the seven days of the documents of the Darwāla Shafa) [35] Sina hospital in history [36] Mashhad city guide [37] Mirdamad grave (View from old Mashhad)[38] Asadi house and Goharshad Ghiyam [39] National library and archives of Iran. Accession No. 293001905 [40] Astan Qods Razavi in documents 
قرارى است كه قلمى شده در ذيل و ظهَر: ... موقوفات عتيقى مزارع

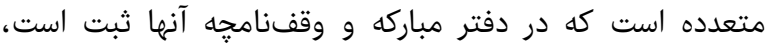

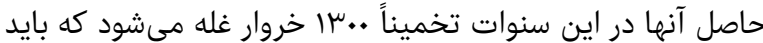

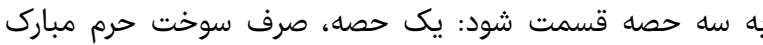

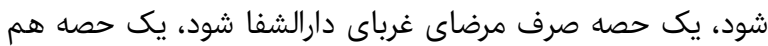

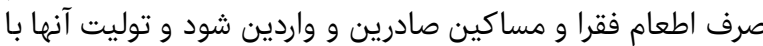

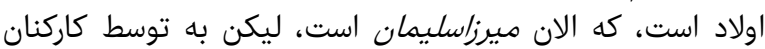

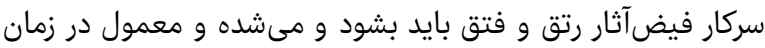

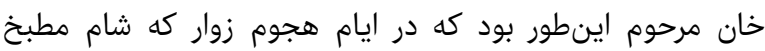

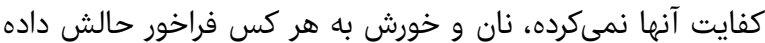

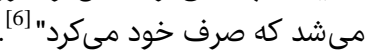

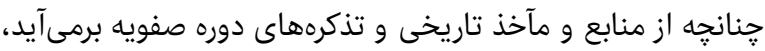

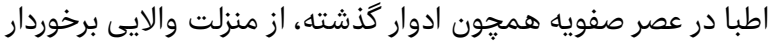

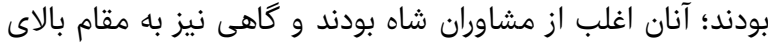

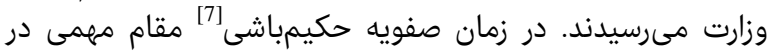

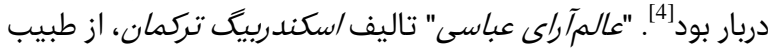

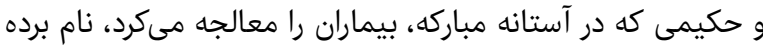

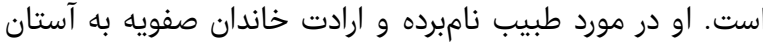

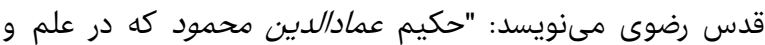

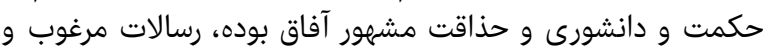

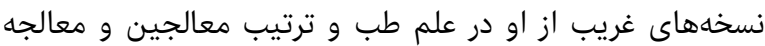

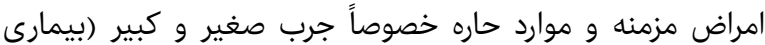

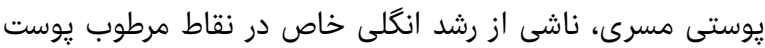

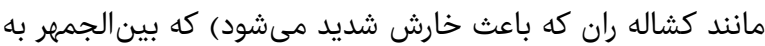

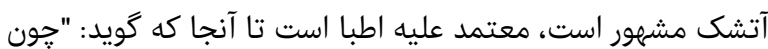

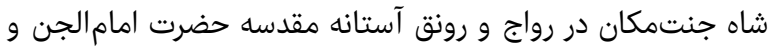

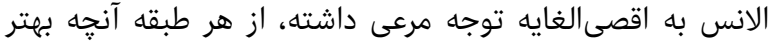

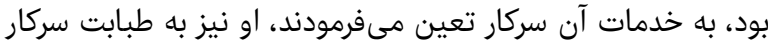

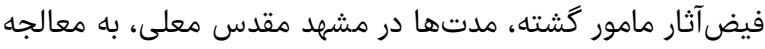

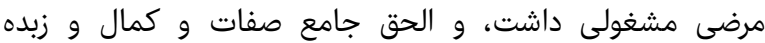
اصحاب كزيده و ارباب افضال بود"[7].

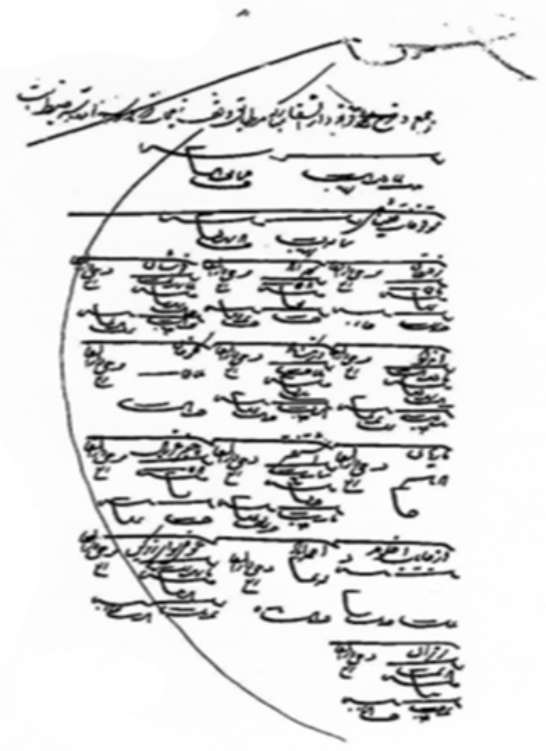

تصوير () سند مربوط به موقوفاتى كه به مصزف تامين هزينههاى دارالشفا مىرسيده است

\section{دارالشفاى آستان قدس رضوى يِيشگام در درمان}

\author{
MSc "على كريميان \\ مركز آموزش مديميان مديت دولتى، تهران، ايران
}

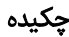

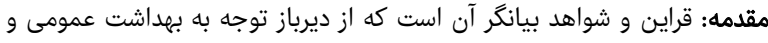

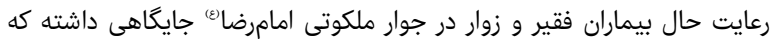

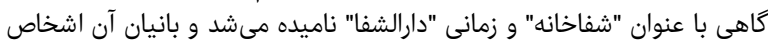

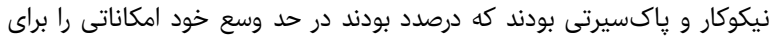

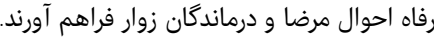

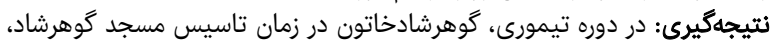

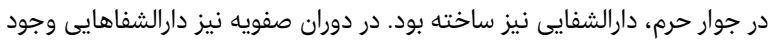

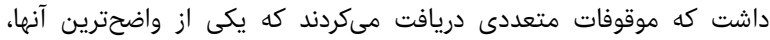

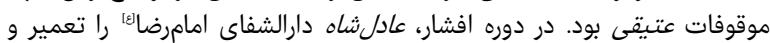

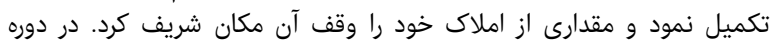

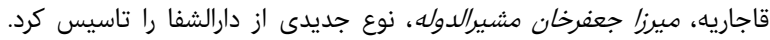

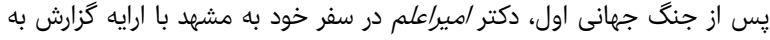

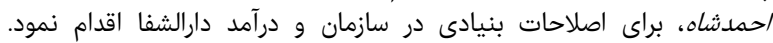

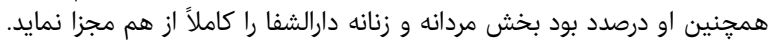

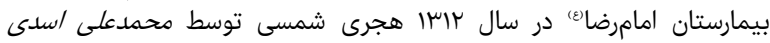

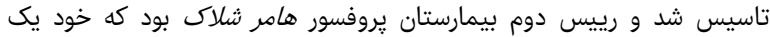

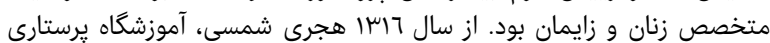

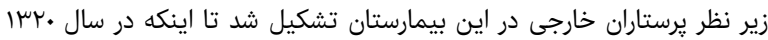

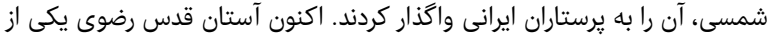

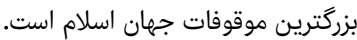

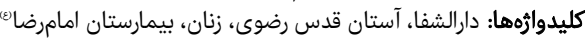

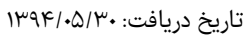

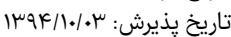

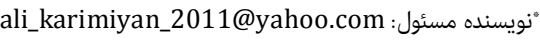

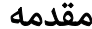

تاريخجه دارالشفا

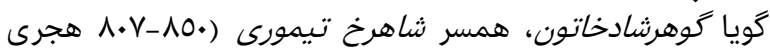

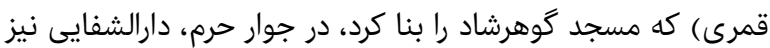

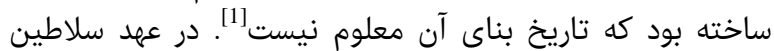

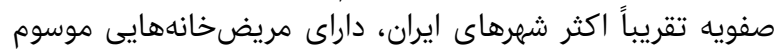

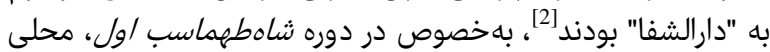

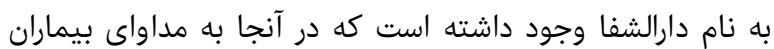

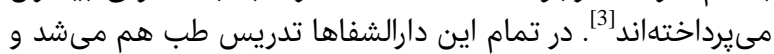

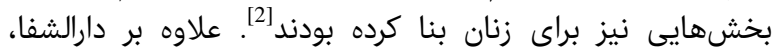

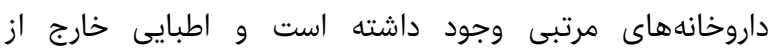

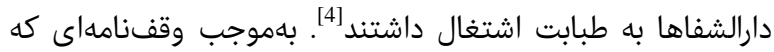

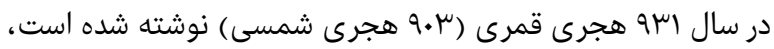

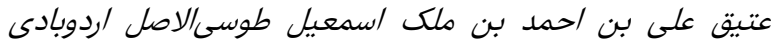

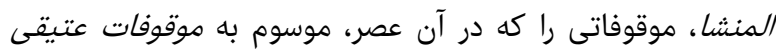

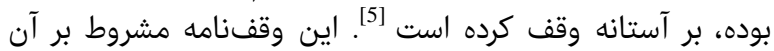
بوده است كه از درآمد موقوفات، سهيمى به كرده مصرف مرضن مرضاى فقير

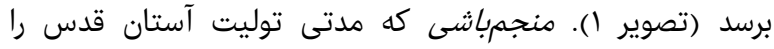

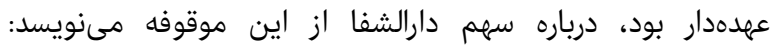

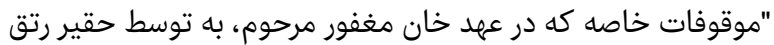

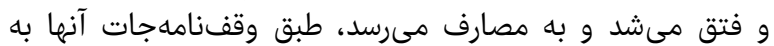




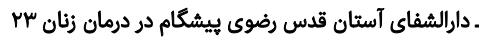

مزبور رسانيده، نسخه بر كميت و كيفيت آن درست داشته به

دفترخانه همايون رسانند"[11].

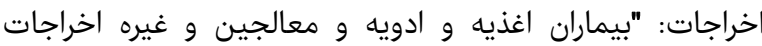

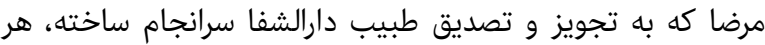

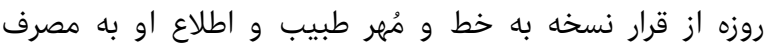

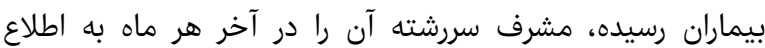

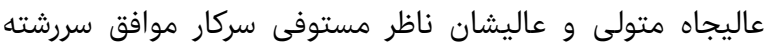

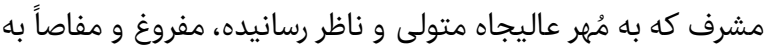

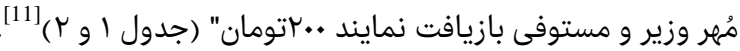
جدول () مصارف نقدى و جنسى، مواجب اطبا، بيماردار، ناظران اغذيه، ادويه و

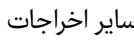

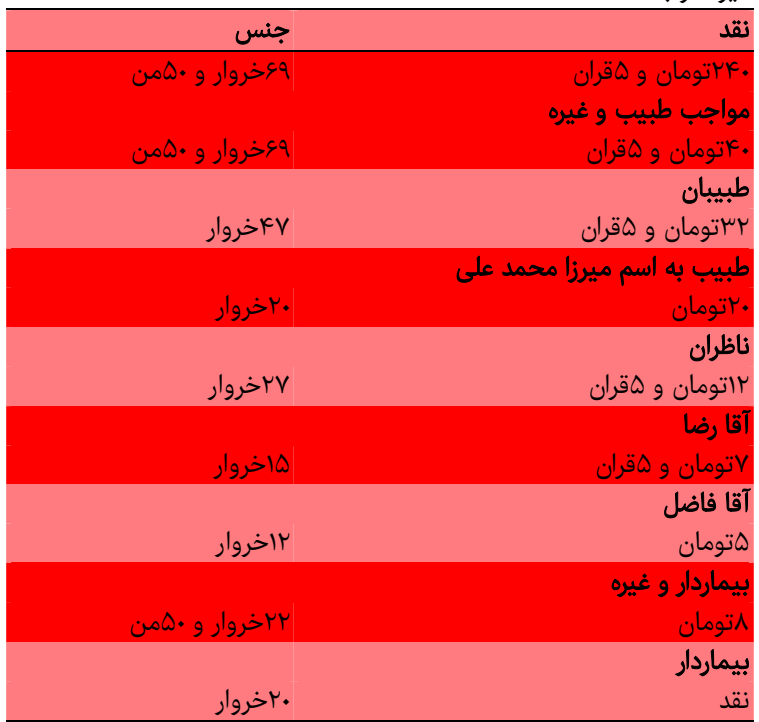

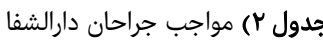

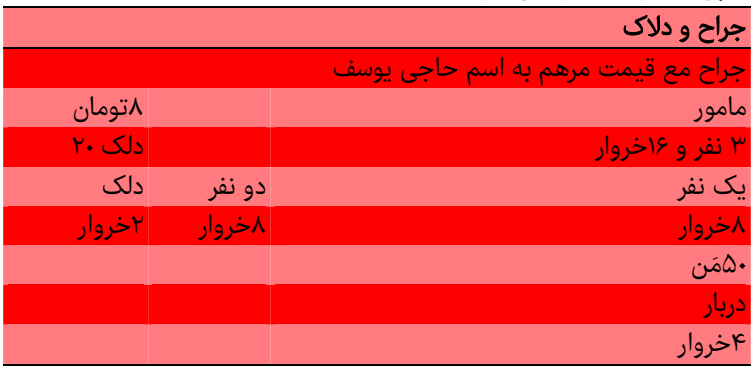

دارالشفا در دوره قاجاريه

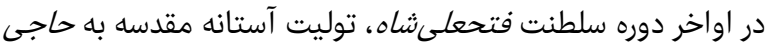

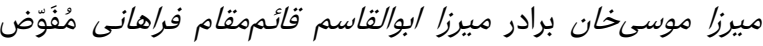

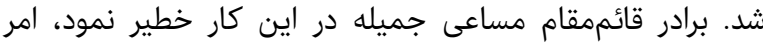

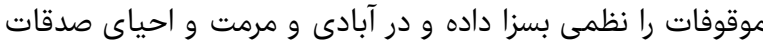

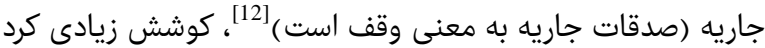

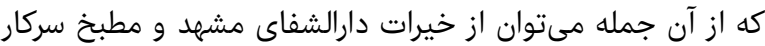

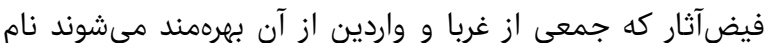

برد [13.

مشرف در تشكيلات آستان قدس كه به معنى ناظر بوده است، بر

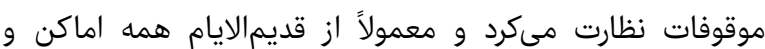

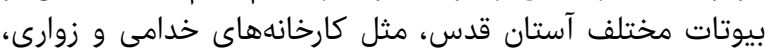

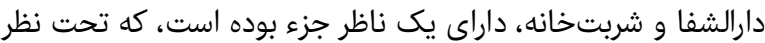

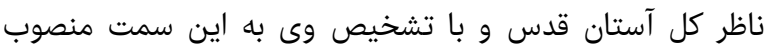

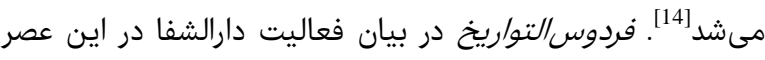

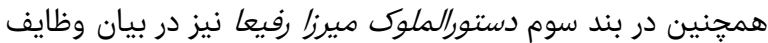

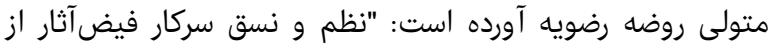

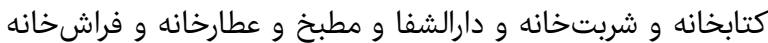

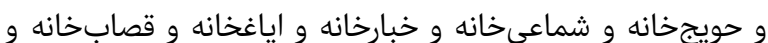

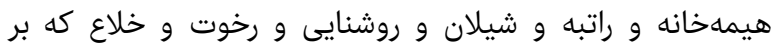

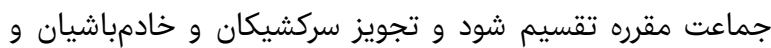

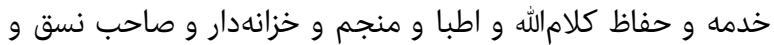

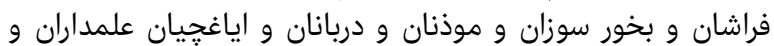

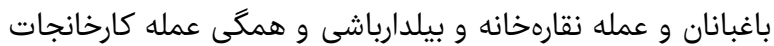

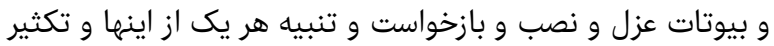

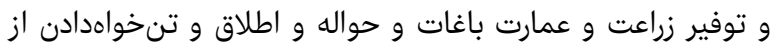

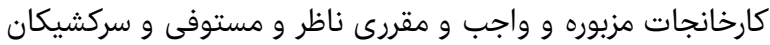

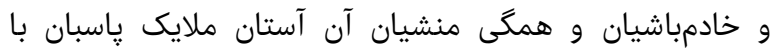

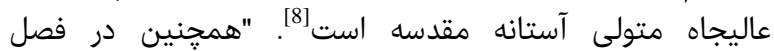

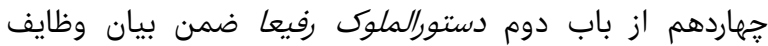

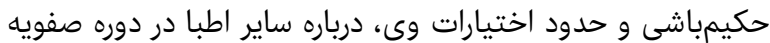

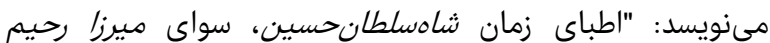

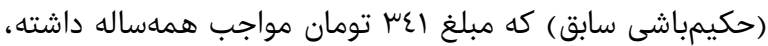

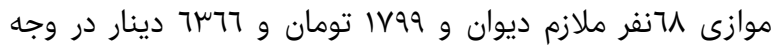

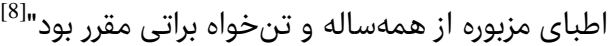

دارالشفا در عصر افشار

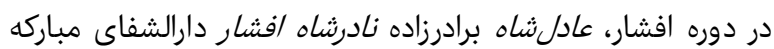

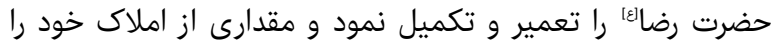

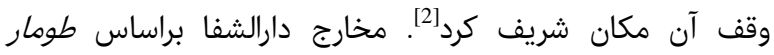

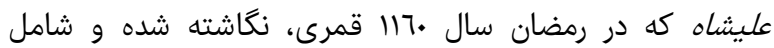

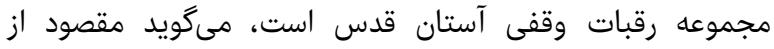

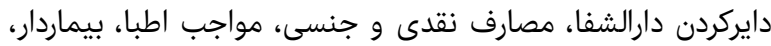

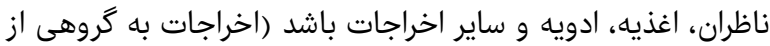

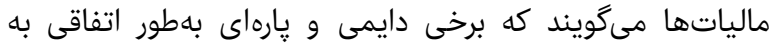

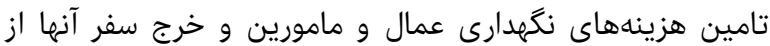

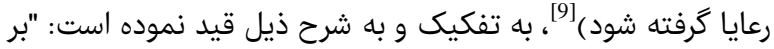

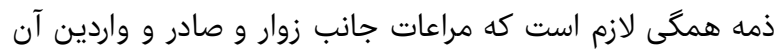

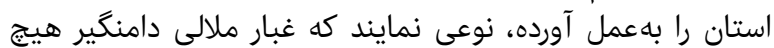

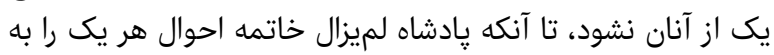

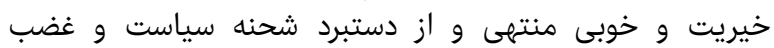

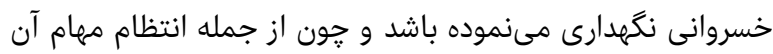

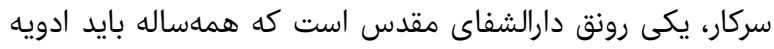

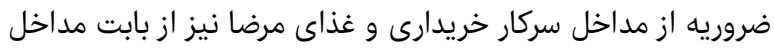

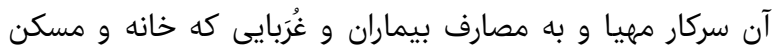

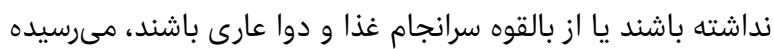

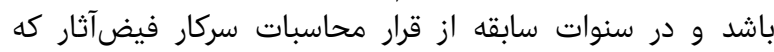

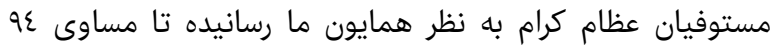

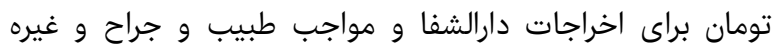

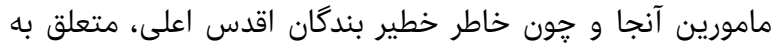

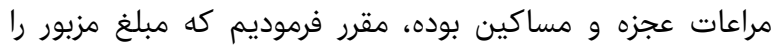

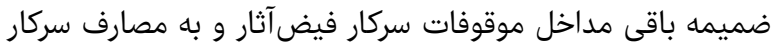

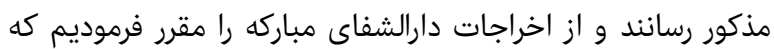

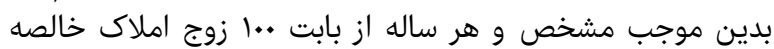

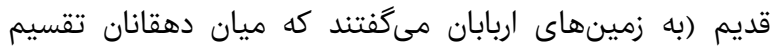

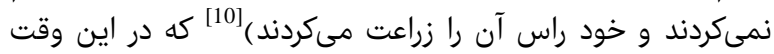

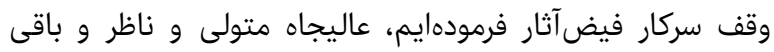

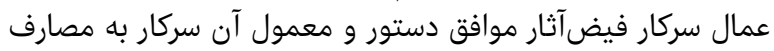


جعفرخان در عهد توليت خودش در خيابان بالا، دارالشفاى خوبى

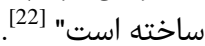

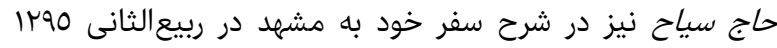

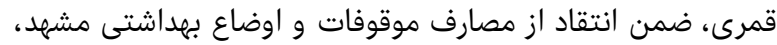

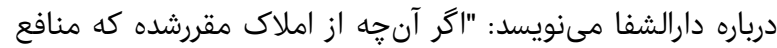

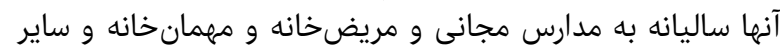

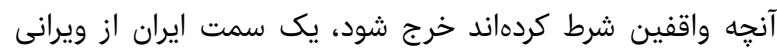

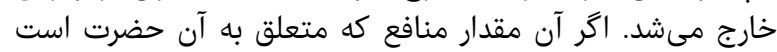

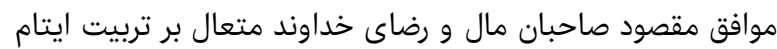

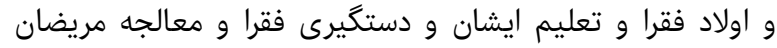

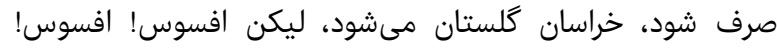

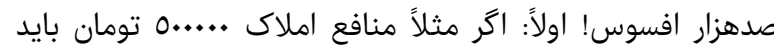

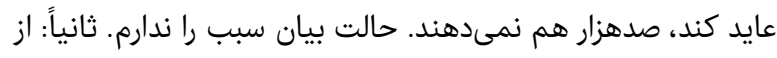

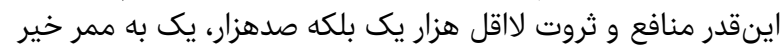

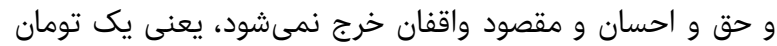

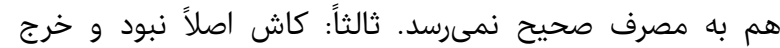

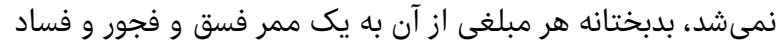

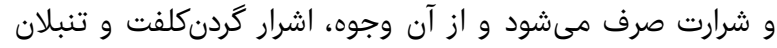

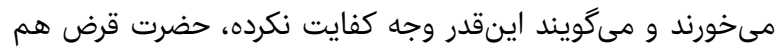

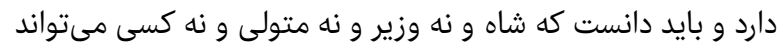

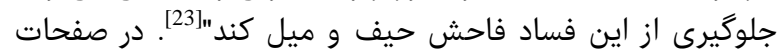

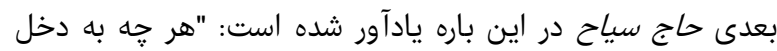

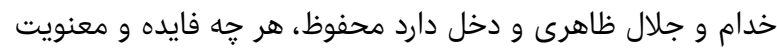

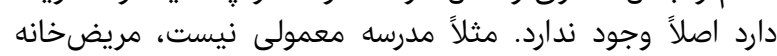

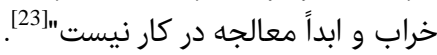

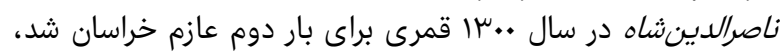

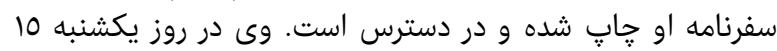

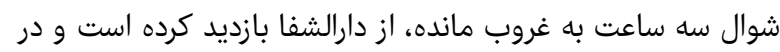

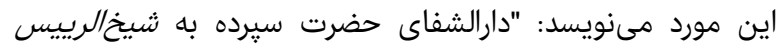

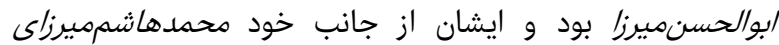

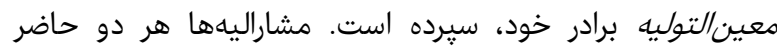

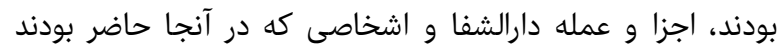

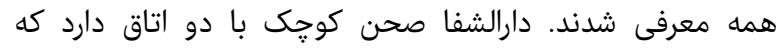

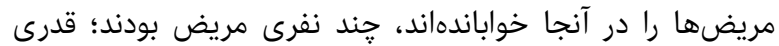

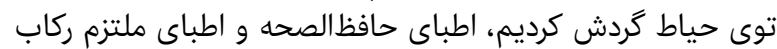

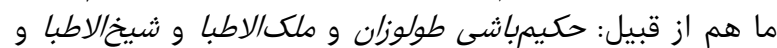

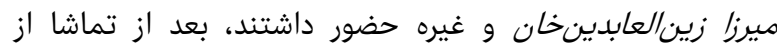

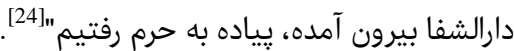

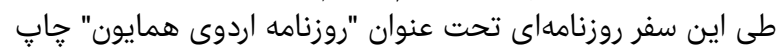

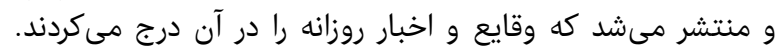

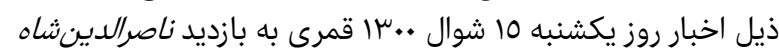

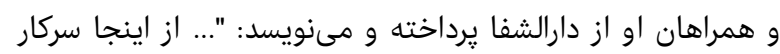

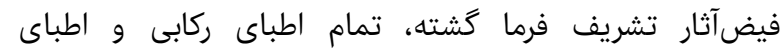

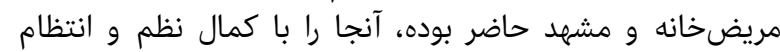

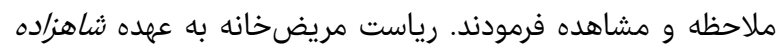

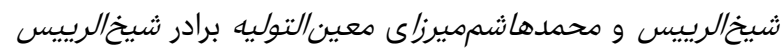
محول است، هر دو حاضر و مورد مراحم ملوكانه شده، از از آنجا به معاريه

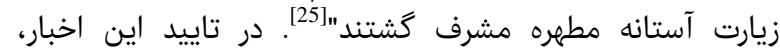

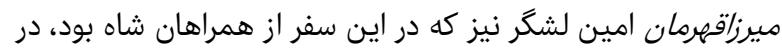

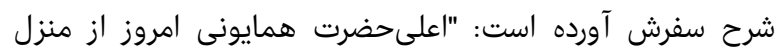

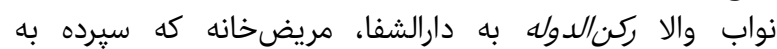

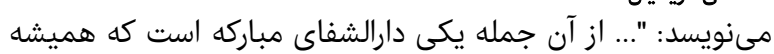

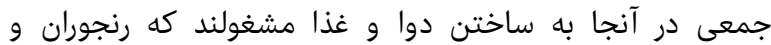

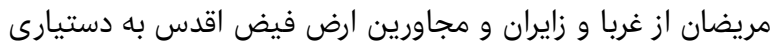

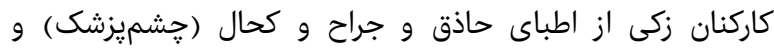

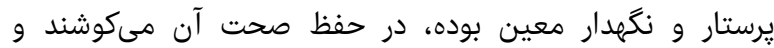

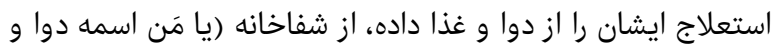

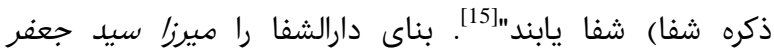

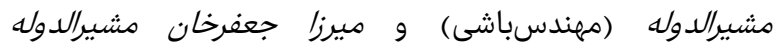

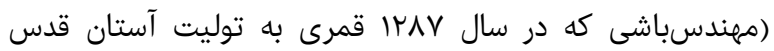

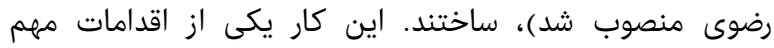

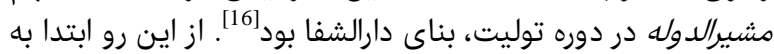

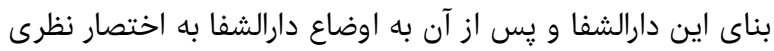

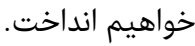

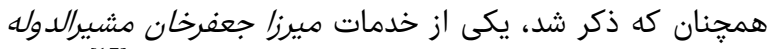

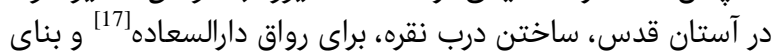

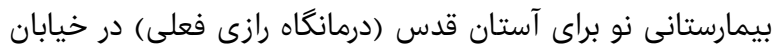

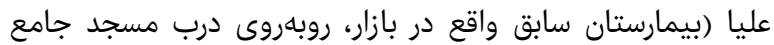

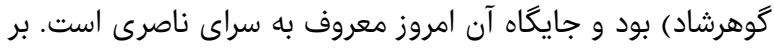

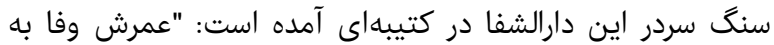

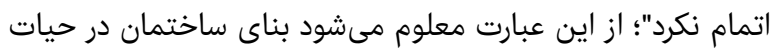

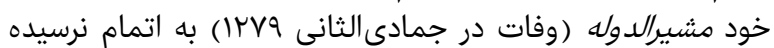

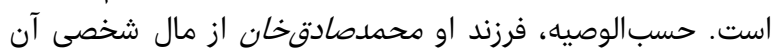

مرحوم اين بنا را به اتمام رسانيد [18].

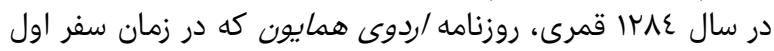

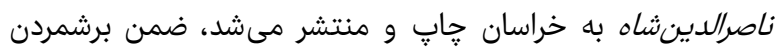

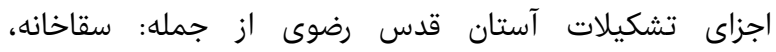

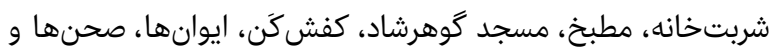

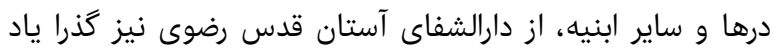

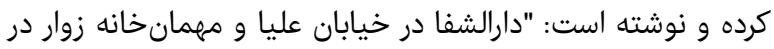

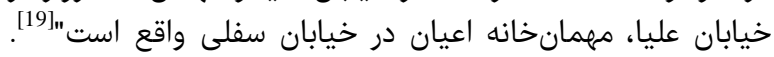

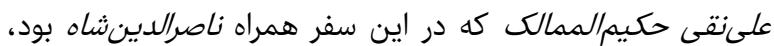

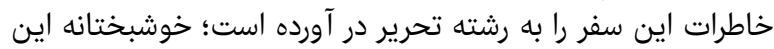

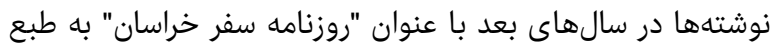

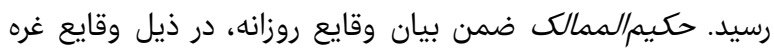

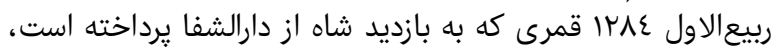

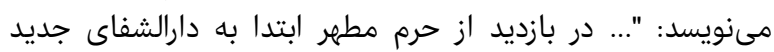

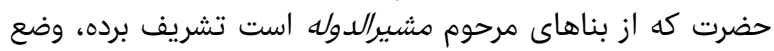

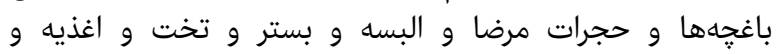

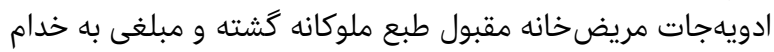

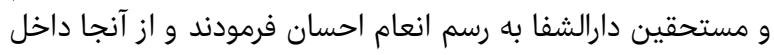

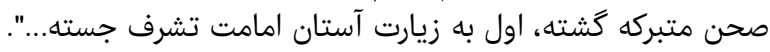

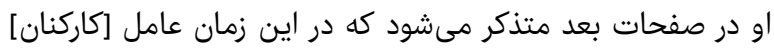

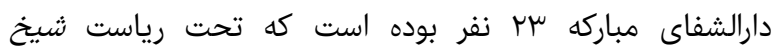

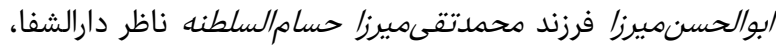
مشغول به كار بودهاند [20] فركاند

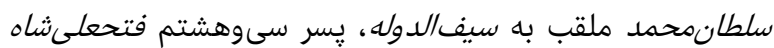

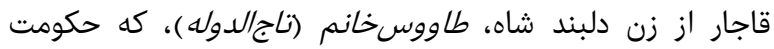

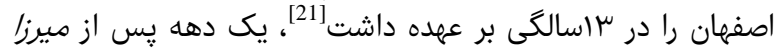

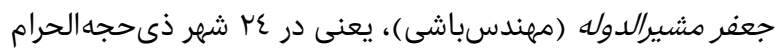

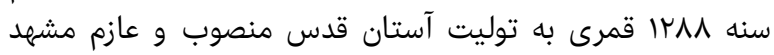
شد. او در خاطراتش از رونق دارالشفا ياد مىكند: "مرحوم ميرزا 
ra

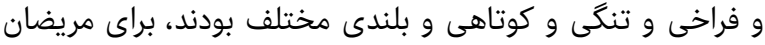

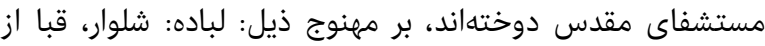

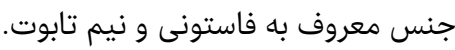

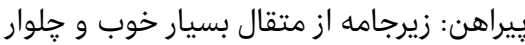

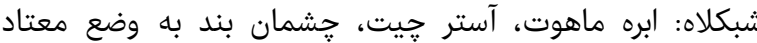

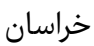
كفش: جتنك خراسانى در نهايت راحت و محكم و ظريف

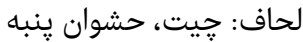
تشك: متقال، حشوان ينبه بان مشان ملحفههاى سفيد كه بعد از

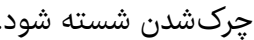
متكا: جيت، حشوان پِنبه با ملحفههاى جيت كه بعد از خركشدن

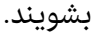
ظروف: از آوانى مالالتجاره مسكوكه از آهن مىسازند و لعابى

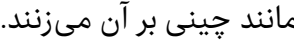

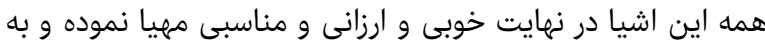

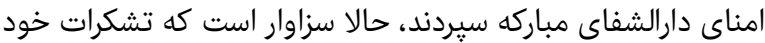

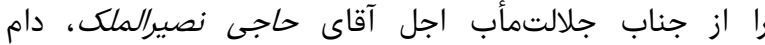

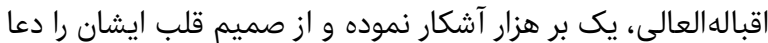

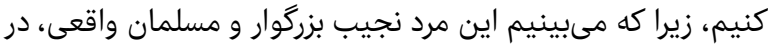

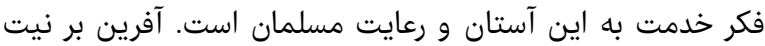

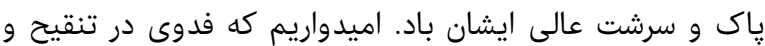

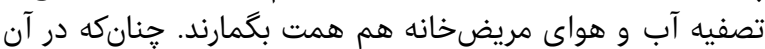

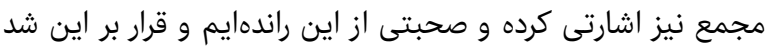

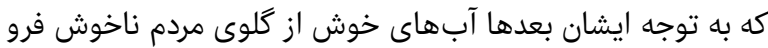

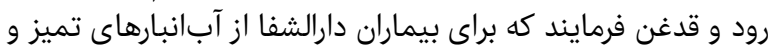

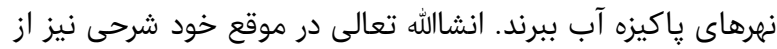

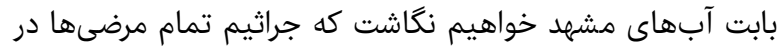

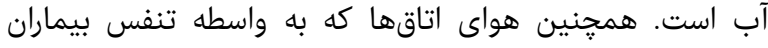

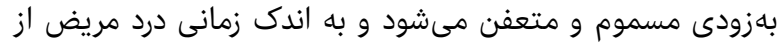

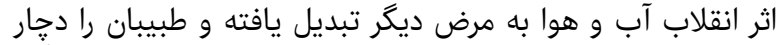

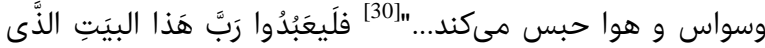

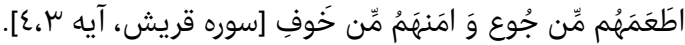

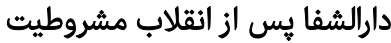

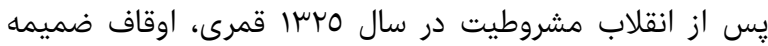

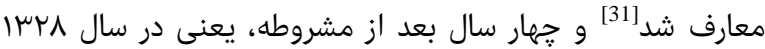

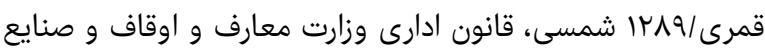

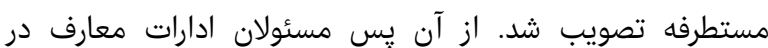

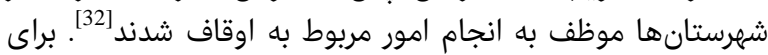

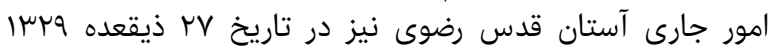

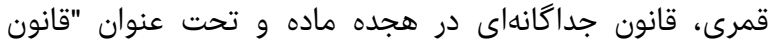

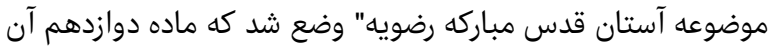

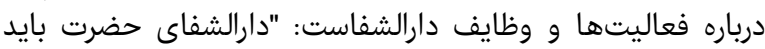

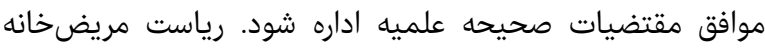

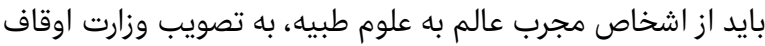

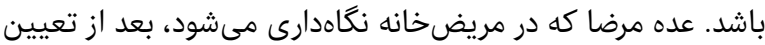

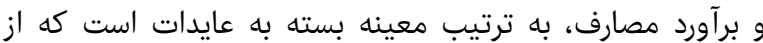
موقوفات مختصه دارالشفا حاصل مى شود" (تصوير ا) [33]

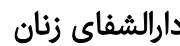
در بررسى مشاغل و مناصب موجنى درد در تشكيلات ادارى دارى دارالشفا به

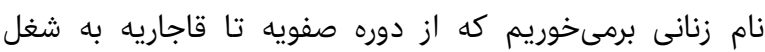

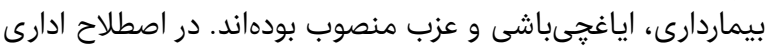

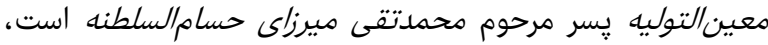

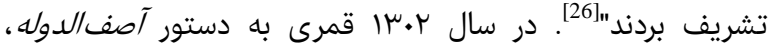

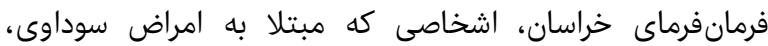

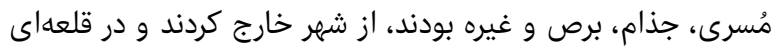

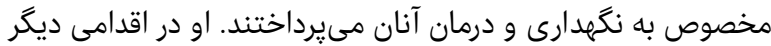

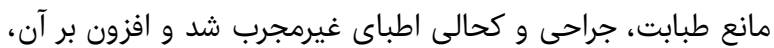

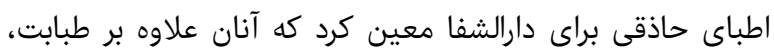

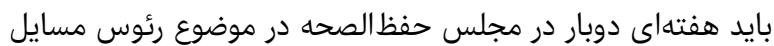

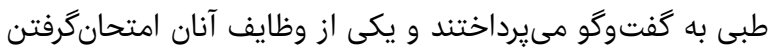

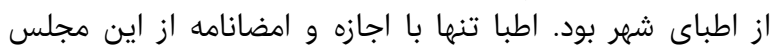

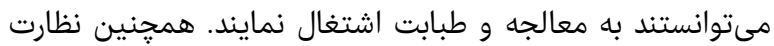

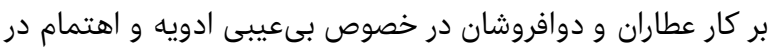

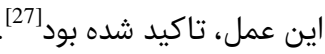

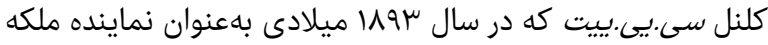

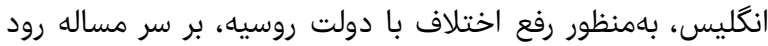

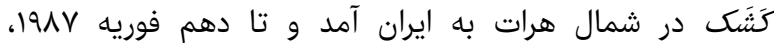

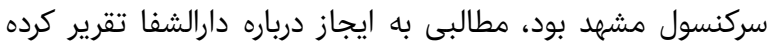

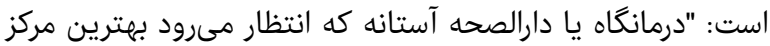

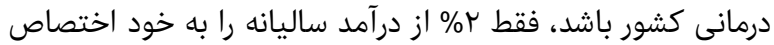

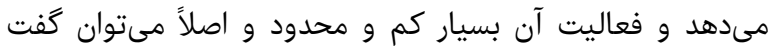

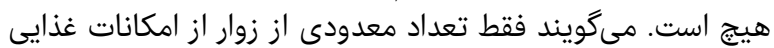

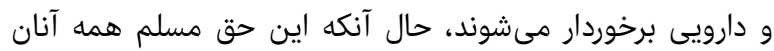

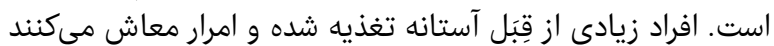

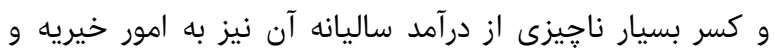

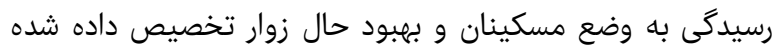

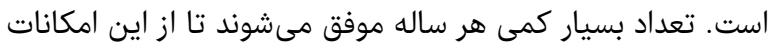
محدود و اندك بهرهمند شوند"[28].

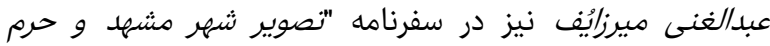

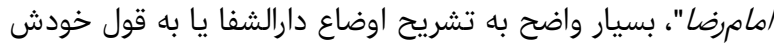

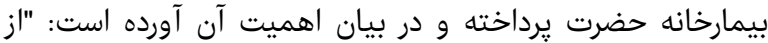

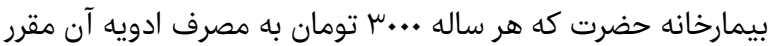

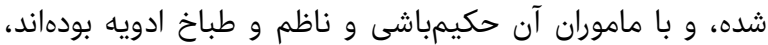

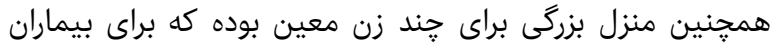

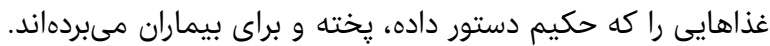

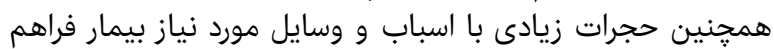

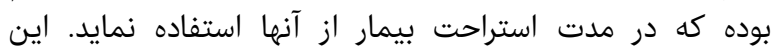

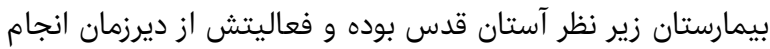

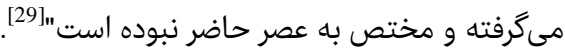
انجمن رسيدگى احوال و لوازم بيماران دارالشفا

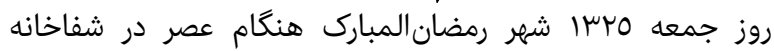

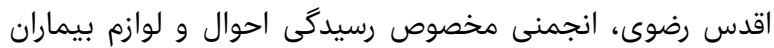

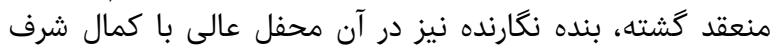

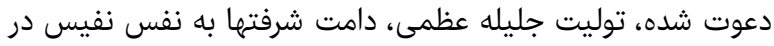
آنجا تشريف داشتند: صفا ديده هاشميان و و فحول امناى آستان دآنان

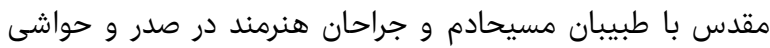

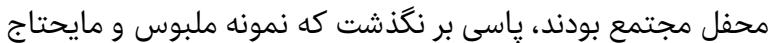

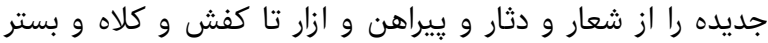

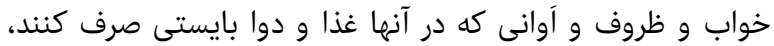

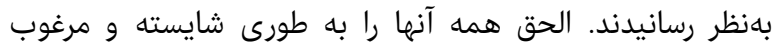

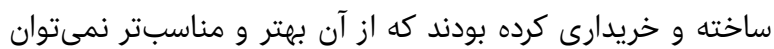

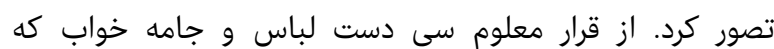
بالضروره برحسب اختلاف اندام و سن بيماران در بزركى و كوجيكى 
را بايد به دارالشفا برسانند، وقتى زمان تاديه مىرسيد، عايدات دجار

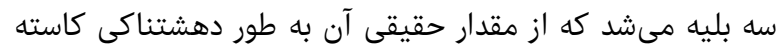

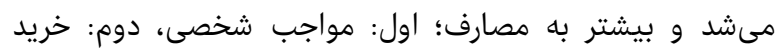

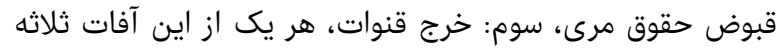

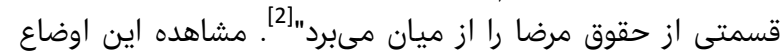

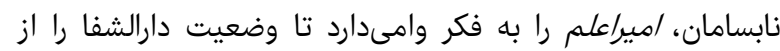

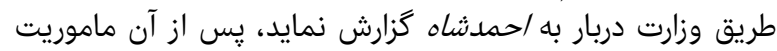

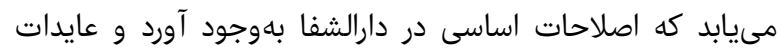

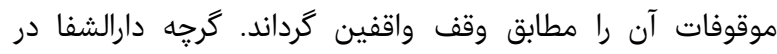

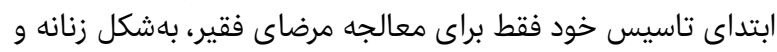

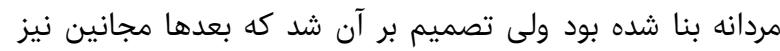

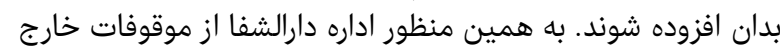

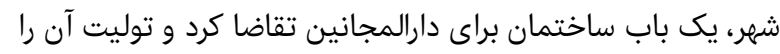

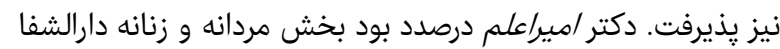

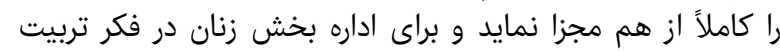

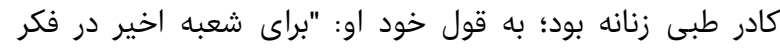

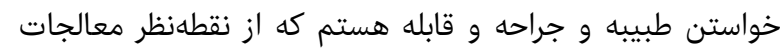

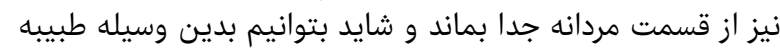

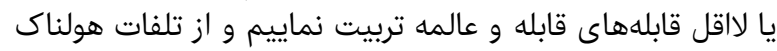

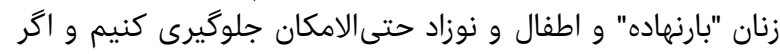

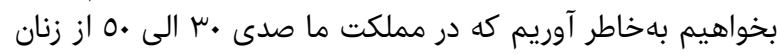

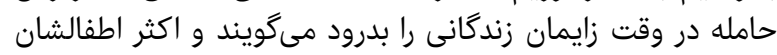

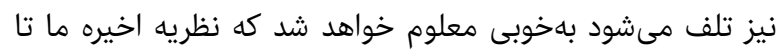

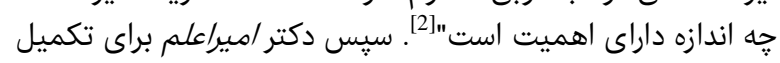

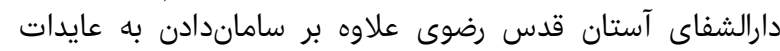

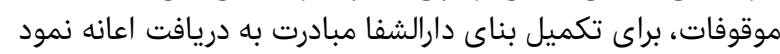

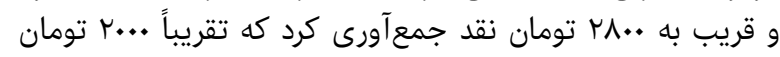

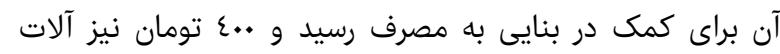

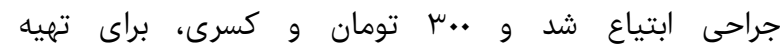

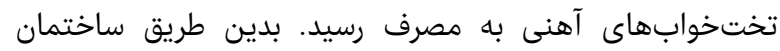

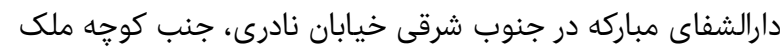

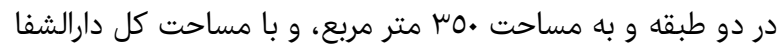

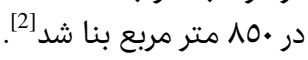

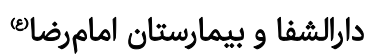

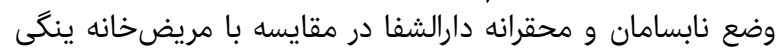

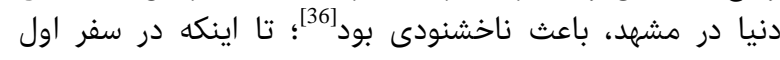

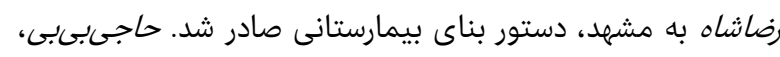

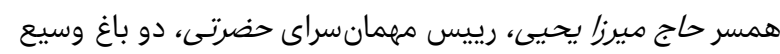

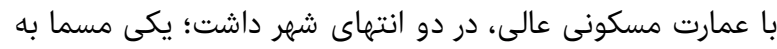

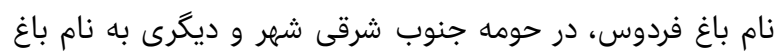

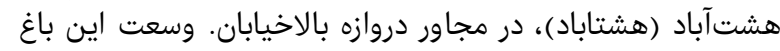

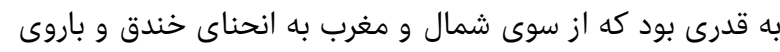

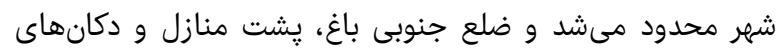

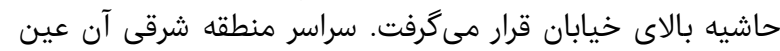

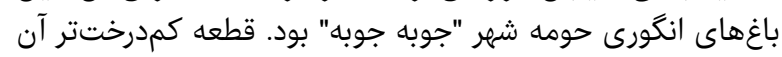

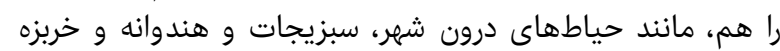

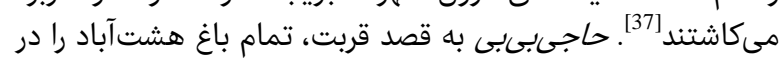

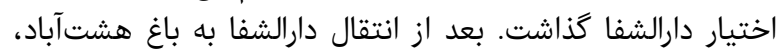

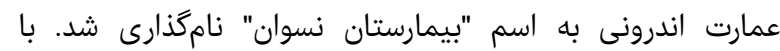

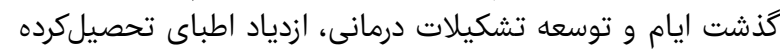

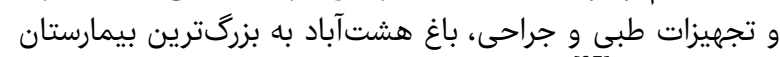

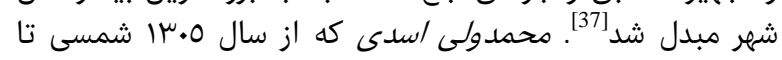

به متصدى بردن مراسلات و وي عيغامهاى مسئولان به كاركنان

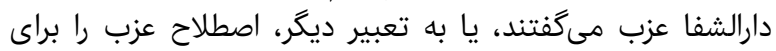

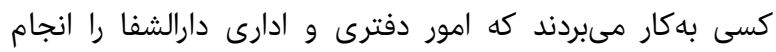

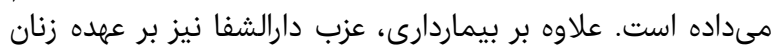

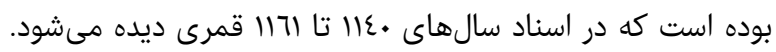

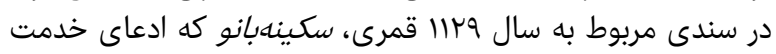

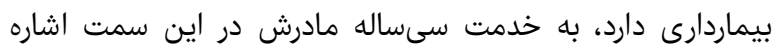

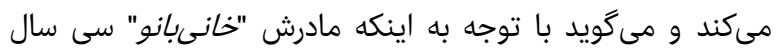

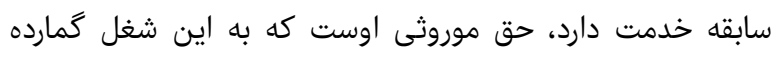

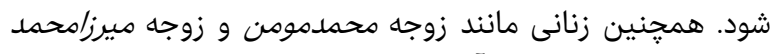

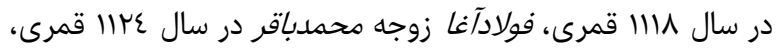

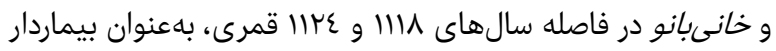

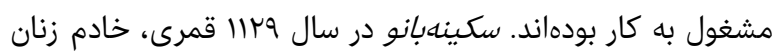

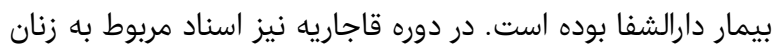

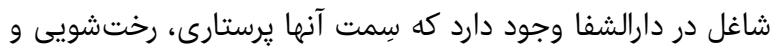

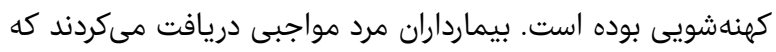

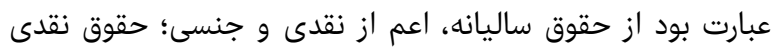

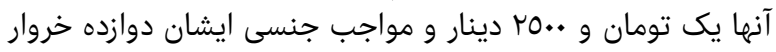

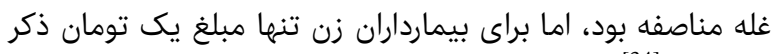

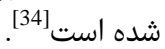

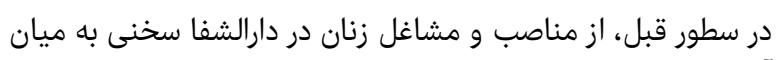

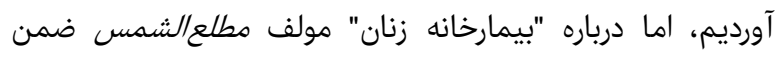

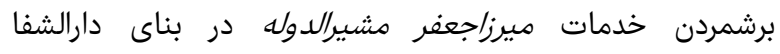

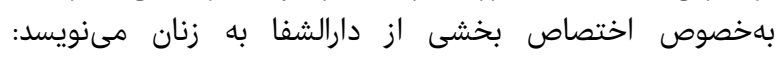

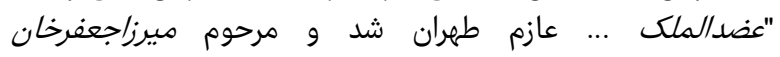

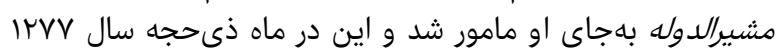

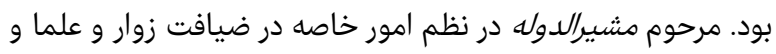

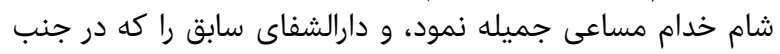

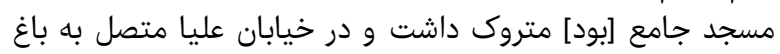

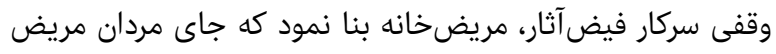

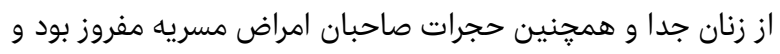

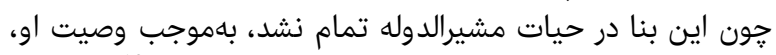

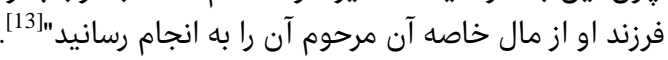

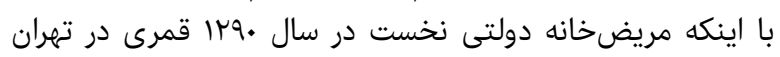

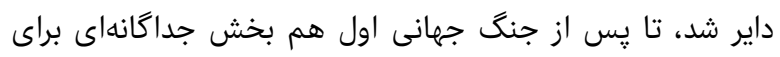

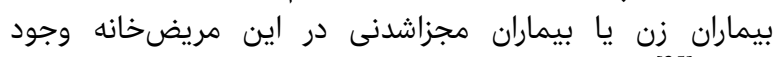

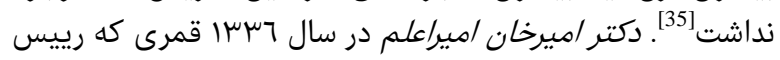

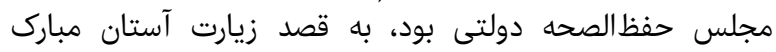

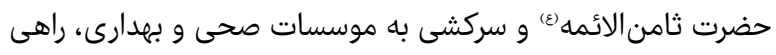

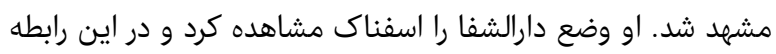

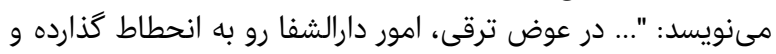

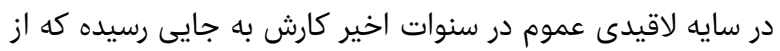

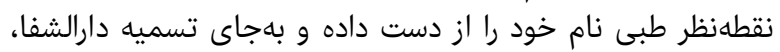

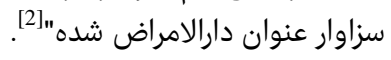

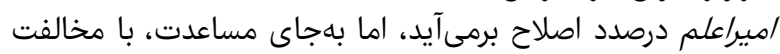

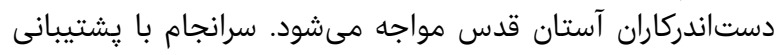

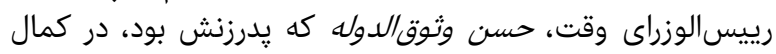

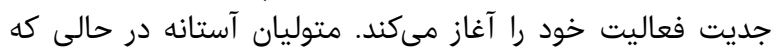

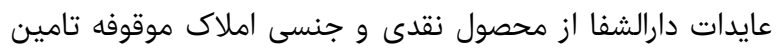

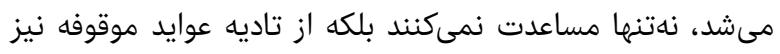

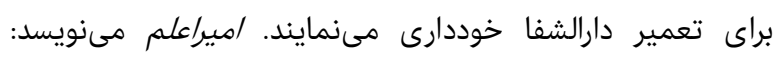
"مستاجران املاك مذكوره هيجگاه در خيال نبودهاند كه مال الاجاره 


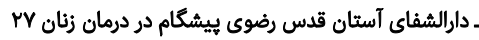

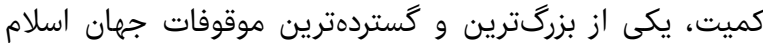

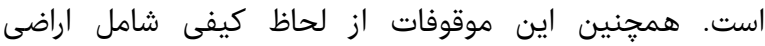

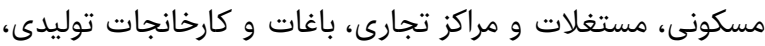

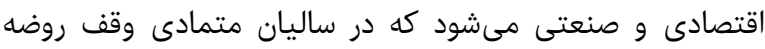

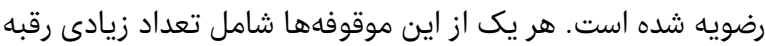

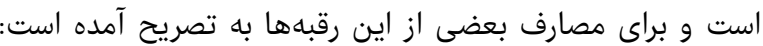

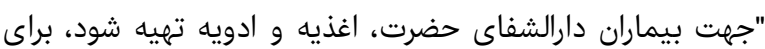

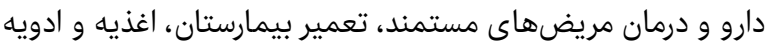

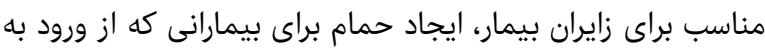

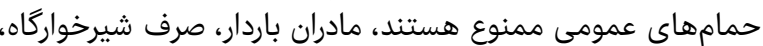

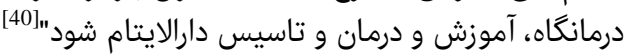

تشكر و قدردانى: موردى از سوى نويسنده گزارش نشده است.

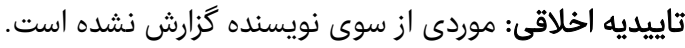

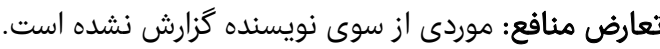

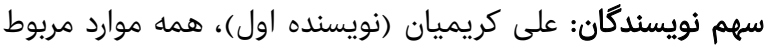

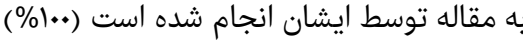
منابع مالى: موردى از سوى نويسنده كزارش اند نشده است است.

منابع

1- Fazel Hashemi SM. Hospital and Dar al-Shafa in the holy shrine of Razavi in the historical mirror. Electronic J Libraries Museums Doc Cent Astan Quds Razavi (Shamseh). 2010;2(7-8):1-23. [Persian]

2- Tashayod AA. Javid Armaghan, or life of the nestes of Dr. Alam A, translator. Teharn: Magazine of Islamic History; 1961. p. 61. [Persian]

3- Tajbakhsh H. History of Iran's Hospital (from beginning to today). Tehran: Institute of Humanities and Cultural Studies; 2000. p. 248. [Persian]

4- Savory R. Iran under the Safavavids. Saba A, translator. Tehran: Tehran book; 1984. p. 61. [Persian]

5- Soozanchi kashani A. Razavi Guest house from Safavid to Ghajar Period (Volume I). Mashhad: Organization of Libraries Museums and Razavi Qods Documents; 2002. p. 199. [Persian]

6- National library and archives of Iran. Accession No. 23525. Tehran: National library and archives of Iran; 2002. p. 73. [Persian]

7- Daneshpazhooh M. Dastoor Al Molouk Mirza Rafia. J Literature Human Tehran Univ. 1969;16:(69-70):540-64. [Persian]

8- Daneshpazhooh M. Dastoor Al Molouk Mirza Rafia va Tazkarat Al Moluok e Mirza Samiea. J Literature Human Tehran Univ. 1968;16:(63-64):475-504. [Persian] 9- Dehkhoda A. Dehkhoda Dictionary (Volume I). Tehran: Tehran University ress; 1994. p. 94. [Persian]

10- Lambton A.K.S. Landlord and peasant in Persia. Amir M, translator. Tehran: Scientific and Cultural Company; 1960. p. 746. [Persian]

11- Mostowfi Hamedani $H$. The face of the sacred endowment Astan Moghaddas Samen Alaemah Ali Ibn Musa al Reza. Mashhad: Anonymous Publisher; 1938. [Persian]

12- Jafari Langeroudi M. Terminology of law. Tehran: Ibn Sina Publication; 1967. p. 406. [Persian]

13- Etemad al Saltaneh M. Matla Al Shams. Borhan Limoudehi T, Hassankhani M, editors. Matla Al Shams: The history of sacred Mashhad, in the history and geography of the famous places of Khorasan. Tehran: Farhangsarsa Publication; 1983. pp. 522-3.[Persian]
ييش از آن كه در دركيرىهاى موسوم به "قيام گوهرشاد" در سال

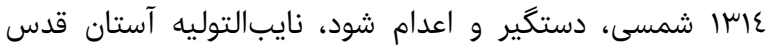

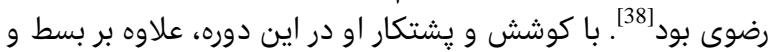

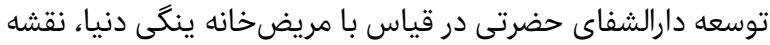

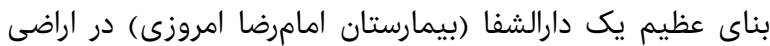

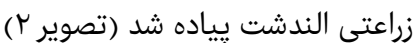

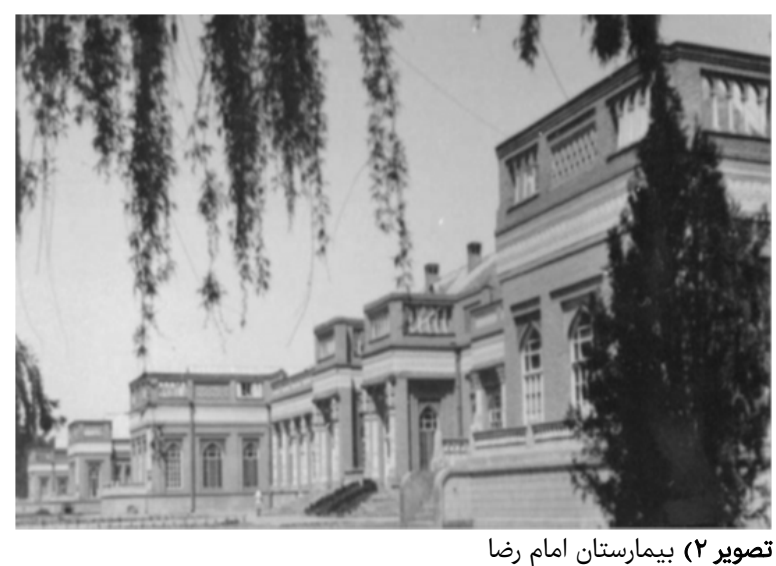

ساخت اين دارالشفا با مساحت .. مIV9 متر مربع با شتاب آغاز شد.

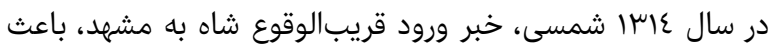

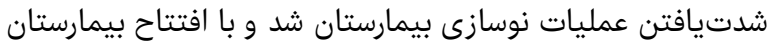

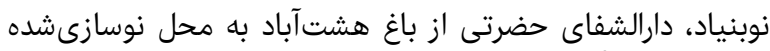

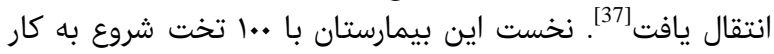

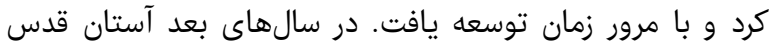

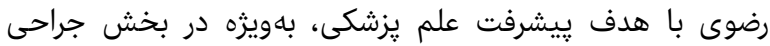

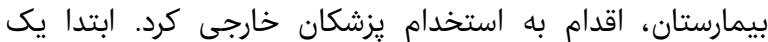

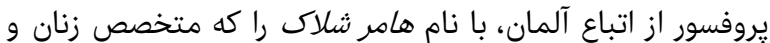

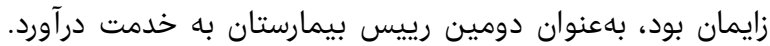

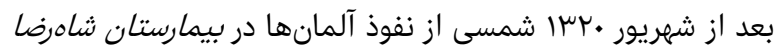

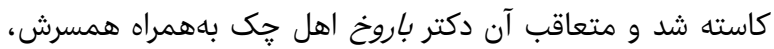

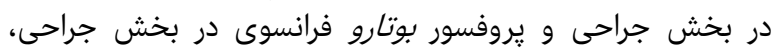

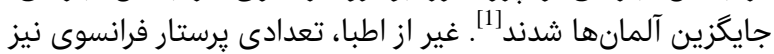

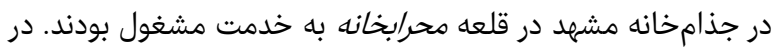

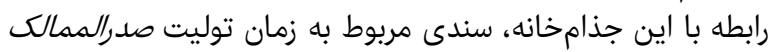

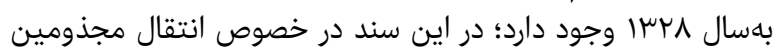

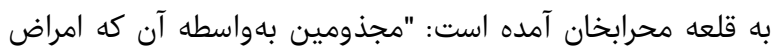

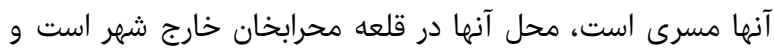

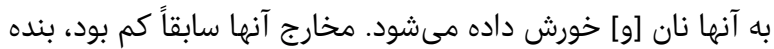

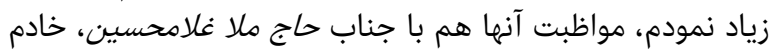

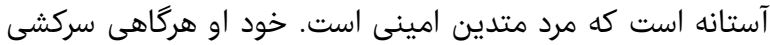

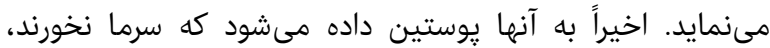

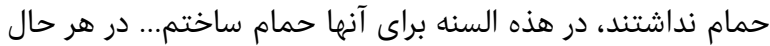

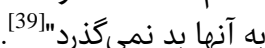

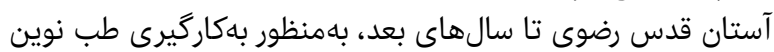

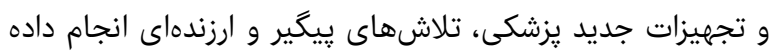

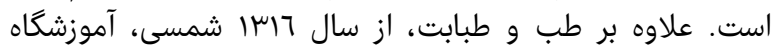

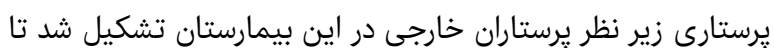

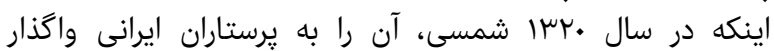

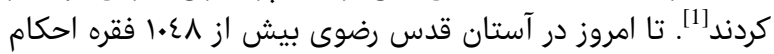

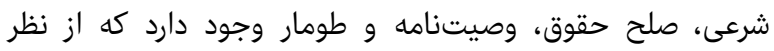


Tehran: Asatir Publication;1995. p. 45. [Persian]

27- Anonimous Writer. Iran newspaper. Tehran. 1885; 4 January. NO. 574. p. 3. [Persian]

28- Yate CE. Khurasan and Sistan. Tehran: Yazdan Publication; 1977. P. 321. [Persian]

29- Taherniya B. Mashhad from the tourists' view from 1600-1914. Mashhad: Astan Qods Razavi Publication; 1997. p. 204. [Persian]

30- Anonimous Writer. Adab newspaper: Tehran. 1902; 6 January. NO. 18. pp. 41-2. [Persian]

31- Foyoozat M. Statement by Mr. Foyoozat: Head of the office of the endowment on endowments. Tehran: Talim va Tarbiat. 1936;91:128-36. [Persian]

32- Salimifar M. A look at Waqf and its economic and social effects. Mashhad: Astan Qods Razavi, Islalmic Research Foundation; 1391. p. 90. [Persian]

33- Karimaiyan A. Astan Qods Razavi foundation after mashroote revolution. Mashhad: Organization of Libraries Museums and Razavi Qods Documents; 2002. p. 41. [Persian]

34- Shahidi H. A Look at traditional medicine in the Dar ul Shafi Astan Quds Razavi in the Safavid age (Review of the seven days of the documents of the Darwāla Shafa). Ganjineh Asnad. 2009;75(22):63-84. [Persian]

35- Tehran University Of Medical Sciences. Sina hospital in history. Tehran: Teymoorzadeh Publication; 1999. p. 16. [Persian]

36- Mofakham Payan L. Mashhad city guide. Mashhad: The geological society (geographic) of Khorasan; 1966. pp. 46-7. [Persian]

37- Baghiei GH. Mirdamad grave (View from old Mashhad). Tehran: Gutenberg Publication; 1994. pp. 124-6. [Persian]

38- Karimian A. Asadi house and Goharshad Ghiyam. Shilan Publication; 2010. pp. 204-8. [Persian]

39- National library and archives of Iran. Accession No. 293001905. Tehran: National library and archives of Iran. [Persian]

40- Karimian A. Astan Qods Razavi in documents. Mah Tarikh va Joghraphiya. 2008;(125):86-96. [Persian] Presentation of Shrine documents and associated places in the Qajar period. Mashhad: Organization of libraries museums and Razavi Qods documents; 2002. p. 123. [Persian]

15- Bastami N. Ferdows al tavarikh. Tabriz: lithography; 1897. [Persian]

16- Karimian A. Permanent memories: Astan Quds dar al Shafa narrates the documents. Moon History Geography Book. 2008;125:86-99. [Persian]

17- Mirza Jafar Khan Mohandesbashi (Mushir Al douleh). Treatise on research on the crossroad. Tehran: Institute Culture of Iran Foundation Publication; 1969. pp. 14-5. [Persian]

18- Mahdi V. Story of the Nawab Toluity of the magnificent Astan Quds Razavi. Namehaye Astan Qods. 1965;(21):86-95. [Persian]

19- Ghasemi SF. Orduye Homayouni newspaper. Tehran: Cultural heritage organization. 2000. P. 52. [Persian]

20- Hakim Al Mamalek A. Khorasan travel newspaper. In: Afshar I, editor. Khorasan travel newspaper Nasser e Din Shah. Tehran: Farhang e Iran Zamin. 1977. p. 259. [Persian]

21- Azad Al Douleh A. Azadi histoty. In: Navaei A, editor. History of the life of Al Imam Abolhassan Ali Ibn Musa al Reza, Mashhad. Tehran: Mahvan Publication; 2000. p. 148. [Persian]

22- Seyf Al Doleh M. Seyf Al Doleh travelogue. In: Khodaparast AA, editor. Seyf Al Doleh travelogue. Tehran: Ney Publication; 1985. p. 318. [Persian]

23- Sayyah Mahalati MA. Memories of Hajj Sayyah. In: Sayyah H, Golkar S, editor. Memories of Hajj Sayyah. Tehran: Amirkabir Publication; 1980. pp. 132-3. [Persian]

24- Naser a Din Shah. Second travelogue of Khorasan. Tehran: Kavosh Va Shabtab Publication; 1984. Pp. 13940. [Persian]

25- Anonimous Writer. Orduye Homayoon newspaper. Tehran. 1883; 12 January. NO. 8. [Persian]

26- Aminlashgar MGh. Khorasan trip newspaper with Naser Al Din Shah. In: Afshar I, Daryrgasht MR, editors. Khorasan trip newspaper with Naser Al Din Shah. 